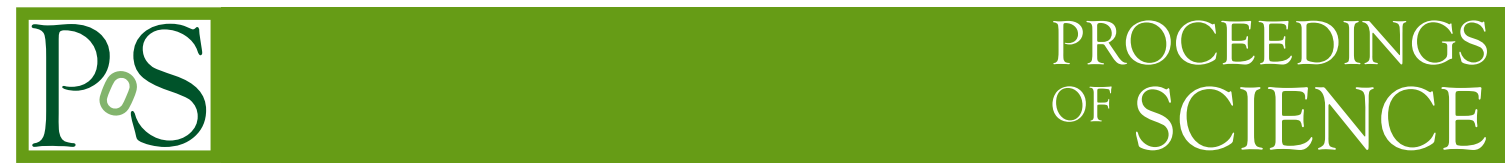

\title{
Measurement of the CKM angle $\gamma$ at LHCb
}

\author{
Marco Gersabeck* \\ University of Glasgow \\ E-mail: marco.gersabeck@cern.ch
}

The precise measurement of the CKM unitarity triangle angle $\gamma$ is a key goal of the LHCb physics programme. The uncertainty on $\gamma$, the currently least-well known of the three angles, will be reduced dramatically. Complementary measurements will be made in tree-level processes, and modes where loop diagrams play an important role. The tree-level measurements will cover time-integrated as well as time-dependent measurements in both the $B_{d}^{0}$ and the $B_{s}^{0}$ sectors. The ensemble of these measurements will provide a powerful test of whether new physics phases contribute to heavy-flavour transitions.

The 2009 Europhysics Conference on High Energy Physics,

July 16 - 222009

Krakow, Poland

*Speaker.

${ }^{\dagger}$ on behalf of the LHCb collaboration 


\section{Introduction}

A new chapter in flavour physics will be opened with the start of data taking at the LHC. The high energy hadron collisions will result in the production of the full spectrum of heavy flavour particles at unprecedented rates. For B-physics this means that in particular the $B_{s}$ sector will be accessible for the first time for high precision measurements. The LHCb experiment specialises in measurements of heavy flavour particles and has been presented in detail [1].

One of the measurements to profit most from the copious heavy flavour particle production at the LHC together with LHCb's measurement capabilities is that of the angle $\gamma$ of the CKM unitarity triangle. So far, direct measurements of $\gamma$ were limited to those exploiting decays of $B_{d}$ mesons. This has left $\gamma$ to be the least well constrained angle of the CKM unitarity triangle.

\section{Measurement of $\gamma$ from Tree-Level Amplitudes}

The decays used to measure the CKM angle $\gamma$ from channels dominated by tree-level amplitudes involve $b-c$ quark transitions. Hence, they are decays $B_{u, d, s} \rightarrow D_{(s)}^{(*)} X$ with $X$ being a pion or kaon [2].

\subsection{Time-Integrated Measurements}

The time-independent approaches of extracting $\gamma$ are based on direct $\mathrm{CP}$ violation measurements in the self-tagging decays $B^{ \pm} \rightarrow D K^{ \pm}$and $B^{0} \rightarrow D K^{* 0}$. Each of the two decay modes can proceed via two diagrams. One of these depends on $V_{c b}$, while the other is CKM suppressed due to its dependence on $V_{u b}$. Furthermore, the latter amplitude is also colour-suppressed for the decay $B^{ \pm} \rightarrow D K^{ \pm}$.

The relative phase between the two respective amplitudes depends on $\gamma$. Additionally, it also depends on the relative strong phase $\delta_{B}$. Another parameter entering the equations is the ratio of the amplitudes of the suppressed and the favoured diagrams, $r_{B}$. The size of $r_{B}$ describes the size of the interference between the amplitudes and is hence related to the sensitivity to $\gamma$. For $B^{ \pm} \rightarrow D K^{ \pm}$ $r_{B}$ is expected to be about 0.1 , while it should be about 0.3 for $B^{0} \rightarrow D K^{* 0}$. Finally, parameters that depend on the $D$ decay used in the reconstruction have to be accounted for as well.

The various methods of extracting $\gamma$ from time-independent measurements differ in the decay channel of the $D$ meson that they use. The GLW method suggests to use $D$ decays into CP eigenstates such as $K^{+} K^{-}$and $\pi^{+} \pi^{-}$[3]. The ADS method exploits $D$ decays into the flavour specific mode $K^{+} \pi^{-}$[4]. This mode introduces an additional phase originating from the strong phase difference between the Cabibbo favoured decay $D^{0} \rightarrow K^{-} \pi^{+}$and the doubly Cabibbo suppressed decay $D^{0} \rightarrow K^{+} \pi^{-}$. LHCb has studied the possibility of combining the GLW and ADS measurements in order to overconstrain the system of phases and amplitudes.

It has further been proposed to extract $\gamma$ from $B^{ \pm} \rightarrow D K^{ \pm}$decays, where the $D$ decays into a three-body final state (GGSZ/B method) [5, 6]. LHCb has studied the Dalitz plot analysis of the decay $D \rightarrow K_{S} \pi^{+} \pi^{-}$. Two methods have been exploited: a model-dependent un-binned fit, and a model-independent binned fit. While the model-dependent fit suffers from large model errors $\left(6^{\circ}-14^{\circ}\right)$, the binned fit requires external input on strong phases, which bring an uncertainty of $2^{\circ}$, and it has potentially worse statistical power. 


\subsection{Time-Dependent Measurements}

For the first time, LHCb will be able to make use of time-dependent measurements in the $B_{s}$ sector to measure $\gamma$. The time-dependent CP asymmetries in the decay $B_{s} \rightarrow D_{s}^{ \pm} K^{\mp}$ measure $\gamma-$ $2 \phi_{M}$ through the interference of the decay $B_{s}^{0} \rightarrow D_{s}^{+} K^{-}$with the oscillated mode $\overline{B_{s}^{0}} \rightarrow D_{s}^{+} K^{-}$[7]. Here, $\phi_{M}$ is the $B_{s}$ mixing phase which can be determined from other measurements at LHCb, e.g. in the decay $B_{s} \rightarrow J / \psi \phi[8]$.

Additionally, $\gamma+2 \beta$ can be determined from measurements of time-dependent $\mathrm{CP}$ asymmetries in the decays $B \rightarrow D^{(*) \pm} \pi^{\mp}$. $\gamma$ can be extracted using the existing precise measurements of $\beta$. A combination with the $B_{s}$ mode using $\mathrm{U}$-spin symmetry has been studied, which removes an eight-fold ambiguity and allows to constrain additional parameters.

The overall sensitivity to $\gamma$ from tree dominated decays after a nominal year of data taking at $\mathrm{LHCb}\left(2 \mathrm{fb}^{-1}\right)$ is expected to be between $4^{\circ}$ and $5^{\circ}$. The exact sensitivity depends on the values of the various other parameters involved. However, due to the combination of many methods, there are no regions of insensitivity in the parameter space.

\section{Measurement of $\gamma$ from Loop Dominated Amplitudes}

The extraction of $\gamma$ from loop dominated decays uses the group of $B_{(s)} \rightarrow h^{ \pm} h^{\prime \mp}$ decays [9], where $h$ is either a pion or a kaon. $\gamma$ is extracted from the time-dependent CP asymmetries of the U-spin related decays $B_{d} \rightarrow \pi^{+} \pi^{-}$and $B_{s} \rightarrow K^{+} K^{-}$[10]. The fit is performed simultaneously to 12 exclusive channels with a total of 68 free parameters. A condition is applied requiring the strong phase $\delta$ to be larger than $90^{\circ}$ to suppress a second solution. Allowing for a U-spin breaking of $20 \%$, a sensitivity to $\gamma$ of $7^{\circ}$ is obtained for a nominal year of data taking at LHCb. Additional constraints on $\gamma$ determined from loop dominated decays are expected from the Dalitz analysis of the decay $B^{ \pm} \rightarrow K^{ \pm} \pi^{+} \pi^{-}$.

\section{References}

[1] A. Golutvin, these proceedings.

[2] M. Adinolfi et al., The tree-level determination of $\gamma$ at LHCb, LHCB-ROADMAP-005, to be published.

[3] M. Gronau and D. London, Phys. Lett. B 253, 483 (1991); M. Gronau and D. Wyler, Phys. Lett. B 265, 172 (1991).

[4] D. Atwood, I. Dunietz and A. Soni, Phys. Rev. Lett. 78, 3257 (1997); D. Atwood, I. Dunietz and A. Soni, Phys. Rev. D 63, 036005 (2001).

[5] A. Giri, Yu. Grossman, A Soffer and J. Zupan, Phys. Rev. D 68, 054018 (2003).

[6] A. Bondar, Proceedings of BINP Special Analysis Meeting on Dalitz Analysis, 24-26 Sep. 2002, unpublished.

[7] R. Aleksan, I. Dunietz and B. Kayser, Z. Phys. C 54, 653 (1992).

[8] M. Calvi, these proceedings.

[9] A. Bates et al., Road map for charmless charged two-body B decays at LHCb, LHCB-ROADMAP-006, to be published.

[10] R. Fleischer, Phys. Lett. B 459 (1999) 306 [arXiv:hep-ph/9903456]. 